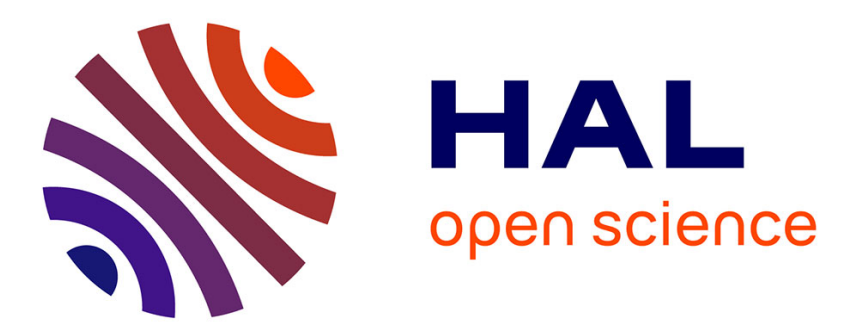

\title{
Diameter-selective non-covalent functionalization of carbon nanotubes with porphyrin monomers.
}

F Vialla, G Delport, Y Chassagneux, Ph Roussignol, Jean-sébastien Lauret, C Voisin

\section{- To cite this version:}

F Vialla, G Delport, Y Chassagneux, Ph Roussignol, Jean-sébastien Lauret, et al.. Diameter-selective non-covalent functionalization of carbon nanotubes with porphyrin monomers.. Nanoscale, 2016, 8, pp.2326. 10.1039/C5NR08023A . hal-01250180

\section{HAL Id: hal-01250180 \\ https://hal.science/hal-01250180}

Submitted on 4 Jan 2016

HAL is a multi-disciplinary open access archive for the deposit and dissemination of scientific research documents, whether they are published or not. The documents may come from teaching and research institutions in France or abroad, or from public or private research centers.
L'archive ouverte pluridisciplinaire HAL, est destinée au dépôt et à la diffusion de documents scientifiques de niveau recherche, publiés ou non, émanant des établissements d'enseignement et de recherche français ou étrangers, des laboratoires publics ou privés. 


\title{
Diameter-selective non-covalent functionalization of carbon nanotubes with porphyrin monomers.
}

\author{
F. Vialla, ${ }^{1}$ G. Delport, ${ }^{2}$ Y. Chassagneux, ${ }^{1}$ Ph. Roussignol, ${ }^{1}$ J.S. Lauret,${ }^{2}$ and C. Voisin ${ }^{1}$ \\ ${ }^{1}$ Laboratoire Pierre Aigrain, École Normale Supérieure, \\ Université P. et M. Curie, Université Paris Diderot, CNRS, 75005 Paris, France \\ ${ }^{2}$ Laboratoire Aimé Cotton, CNRS, École Normale Supérieure de Cachan, Universite Paris Sud, 91405 Orsay, France
}

\begin{abstract}
We report on the spontaneous noncovalent functionalization of carbon nanotubes with hydrophobic porphyrin molecules in micellar aqueous solution. By monitoring the species concentrations with optical spectroscopies, we can follow the kinetics of the reaction and study its thermodynamical equilibrium as a function of the reagent concentrations. We show that the reaction is well accounted for by a cooperative Hill equation, reaching a molecular coverage close to a compact monolayer for a porphyrin concentration larger than a diameter-specific threshold concentration. The equilibrium constant is measured for 16 nanotube chiral species. The Gibbs energy of the reaction (of the order of $-40 \mathrm{~kJ} / \mathrm{mol}$ ) and its evolution with the nanotube diameter is consistent with theoretical calculations of the binding energy. This thermodynamical study shows a strong preferential binding of TPP molecules to larger diameter nanotubes. This original curvature selectivity can be used to induce diameter selective species enrichment.
\end{abstract}

\section{INTRODUCTION}

Carbon nanotubes (NTs) are original nanocarbon materials with attractive mechanical, electronic, optical and thermal properties. Owing to their unique single atomiclayer structure, the chemical functionalization of their sidewall is a valuable handle to deeply engineer their physical properties by making use of the versatility of organic chemistry. In the $\pi$-stacking approach, the conjugated core of the organic molecules mildly interacts with the $\pi$ orbitals of the NT. This scheme is extremely promising since it mostly preserves the intrinsic electronic properties of the nanocarbon while introducing a strong interaction with the ligand, which ensures a good chemical stability. For instance, by using dye molecules it is possible to tune the light absorption and emission features of the compounds in view of bio-labeling or lightharvesting applications [1-4]. In particular, hybrid compounds of NTs functionalized with free-base tetraphenylporphyrin monomers (TPP) show remarkable photophysical properties with highly efficient energy transfer for all chiral species of nanotubes [5, 6]. Nevertheless, a consistent understanding of the supramolecular compound formation and stability is still missing, though highly desirable to elaborate better molecular designs and synthesis protocols.

In this paper, we report on the kinetics and equilibrium properties of the noncovalent binding reaction of TPP monomers (Figure 1d) onto single-wall nanotubes encapsulated in cholate micelles. We bring evidence for the completion of a compact monolayer of TPP monomers on the nanotube sidewall with reaction constants in good agreement with recent calculations [7]. In contrast to previous studies reporting the wrapping of NT into sophisticated polymers $[8,9]$, the general significance of this study lies in the structural simplicity of the ligand and in the generic interaction involved in the functionalization
( $\pi$-stacking). Even with such a small and symmetrical molecule, we observe an original and striking NT diameter dependence in the adsorption affinity that opens the way to easy, reversible diameter-selective functionalization. Finally, we show that one can make use of this selectivity to achieve efficient diameter selective enrichment of the nanotube mix with the help of ultra-centrifugation.

\section{EXPERIMENTAL RESULTS}

\section{Methods and materials}

All the hydrophobic species - NTs and TPP monomers - involved in this study are dispersed in water using sodium cholate. In fact, such bile salt surfactants are known and widely used to yield effective individualization of NTs with a large stability over time $[10-12]$. The stock HiPCO nanotubes solution is made from a commercial nanotube powder (Nanoledge) incorporated at a concentration of a few tenths of $\mathrm{mg} / \mathrm{mL}$ in an aqueous pH8-buffer solution with $2 \%$ wt sodium cholate. The mix is sonicated with an ultrasonic tip for 1.5 hour at $4{ }^{\circ} \mathrm{C}$ and then ultracentrifugated for 1 hour at $120000 \mathrm{~g}$. A stock micelle suspension of TPP solution is obtained from a mix between a similar aqueous pH8-buffer solution with sodium cholate and a high concentration solution of TPP in dichloromethane $(1 \mathrm{mg} / \mathrm{mL})$ in a relative proportion of 10:1. The immiscible phases are mixed together using an ultrasonic tip at $12{ }^{\circ} \mathrm{C}$ until complete evaporation of the organic phase. Different dilutions of this stock solution are used in the titration protocol. The same surfactant wrapping scheme is used to solubilize the non-covalently functionalized compounds based on NTs (Figure 1e) and to increase their stability up to several months $[13,14]$.

The kinetics of the functionalization reaction is monitored by using the specific spectroscopic features of both 
the TPP molecule and the nanotube and of their changes upon functionalization by means of photoluminescence (PL) and absorption spectroscopies. Absorbance spectra are obtained with a commercial 2-channel spectrometer (Perkin-Elmer Lambda 900). Photoluminescence excitation (PLE) maps are obtained with a home-made setup in a 90 degree geometry, where excitation is provided by a monochromator-filtered Xe lamp (spectral full-width at half-maximum of $5 \mathrm{~nm}$ ). The detection is achieved with a $30 \mathrm{~cm}$ spectrograph coupled to an InGaAs detector (Princeton Instruments OMA V).

To assess the thermodynamical parameters of the reaction, we studied the equilibrium composition of a set of solutions obtained by mixing fixed volumes of a stock NT solutions with TPP solutions of increasing concentrations.

\section{Kinetics of the reaction}

The temporal evolution of the absorbance of such a mix of TPP and NT micellar suspensions is presented in Figure 1a,b. The $E_{n, m}^{11}$ optical resonances of the different $(n, m)$ species of carbon nanotubes are observed in the near-infrared window, while the large absorption line at $2.95 \mathrm{eV}$ corresponds to the Soret band of free TPP monomers. A spontaneous evolution is observed within several hours, that can be interpreted as the progressive incorporation of the TPP monomers inside the micelles containing a NT, leading to the functionalization of the NT sidewall. An increasing redshift of the $E_{n, m}^{11}$ transitions of NTs is observed over time (Figure 1a). This shift is interpreted as an increased dielectric screening of the $E_{n, m}^{11}$ excitonic states due to the presence of the TPP in the near vicinity of the tubes [15]. In the same time, the amplitude of the TPP Soret band at $2.95 \mathrm{eV}$ is decreasing, while a new line at $2.82 \mathrm{eV}$ is growing (Figure $1 \mathrm{~b})$. The isobestic point at $2.9 \mathrm{eV}$ reveals the progressive transformation of a species into another, here free monomers of TPP into TPP monomers bound to the nanotube wall. This shift of the absorption spectrum of bound TPP is attributed to the conformational change of the four phenyls in TPP molecules due to the interaction with the NT surface [13].

The absorbance ratio of these two peaks is used to quantitatively monitor the kinetics of the reaction (Figure 1c). A relatively slow kinetics with a time constant of several days is obtained (Figure 1c). This can be understood owing to the fact that the micelles have to merge prior to the interaction between the monomer and the NT, preventing a faster adsorption [16]. This reorganization of the cholate micellar structure is actually associated to a large activation energy of the order of $50 \mathrm{~kJ} / \mathrm{mol}$ [17]. This kinetics can be drastically accelerated, down to a few hours, using conventional methods such as taking one reagent in large excess, soft heating or soft sonication (by placing the spectroscopic cuvettes in a thermo-regulated sonication bath) (Figure 1 (c)).

Interestingly, we can compare this spontaneous mechanism to another functionalization process reported in a previous paper, the so-called micelle swelling method [13]. In this scheme, an organic solvent (dichloromethane) is used as a vector for the hydrophobic monomers to enter the core of the micelles containing the nanotubes by means of ultra-sonication. We note that in the end, all the processes yield suspensions with identical proportions of stacked TPP (Figure 1c), which brings a strong evidence that the thermodynamical equilibrium is reached in all cases.

\section{Thermodynamical equilibrium}

In the following, we focus on the equilibrium state of the suspensions, sampling a wide range of reagent concentrations in order to assess the thermodynamical parameters of the reaction. The functionalization reaction is described with a simple binding mechanism : $C S+T P P \rightleftharpoons C S / T P P$, where $C S$ (Carbon Site) stands for the sites available on the nanotubes, TPP stands for the single TPP monomer and $C S / T P P$ for the filled sites. We make use of the Hill formalism, introducing -for each $(n, m)$ NT species- the reaction constant $K_{n, m}$ and the Hill coefficient $h_{n, m}$. The Hill coefficient accounts for the cooperative nature of the reaction. It is used for instance in the description of the binding of ligands onto proteins [18]. This approach has previously been introduced with success to describe the competition between different wrapping molecules in nanotube solutions $[2,19,20]$. Note that this description ignores the micelle cap for all the reagents and products. In fact, the thermodynamical parameters we actually measure in this experiment are related to the substitution of a surfactant molecule on the nanotube wall by a TPP molecule surrounded by the surfactant shell. However, by changing the nature of the surfactant (we used SDS instead of cholate), we found similarly a spontaneous functionalization with a comparable reaction constant (see SI). Since the structure of those two surfactants is very different, this shows that the reaction is mainly driven by the NT/TPP interaction.

The chemical composition of the suspension at equilibrium can be monitored using the specific photoluminescence signatures of the reagents and products. In particular, since the PL of the TPP molecules is completely quenched once they bind to the nanotube due to ultrafast energy transfer [6], the free TPP concentration can be assessed by comparing the TPP Q band PL intensity to that of a reference TPP micellar suspension of known concentration. In addition, PL excitation measurements (PLE) allow us to single out the different chiral species. Each spot in the PLE map (Figure 2a) corresponds to the 

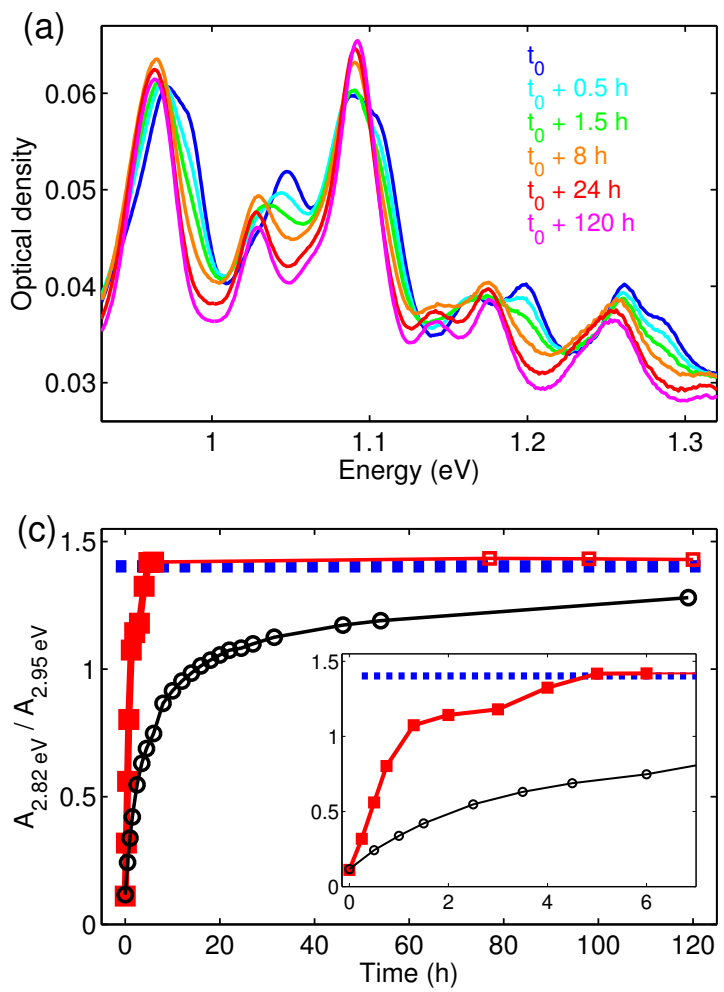

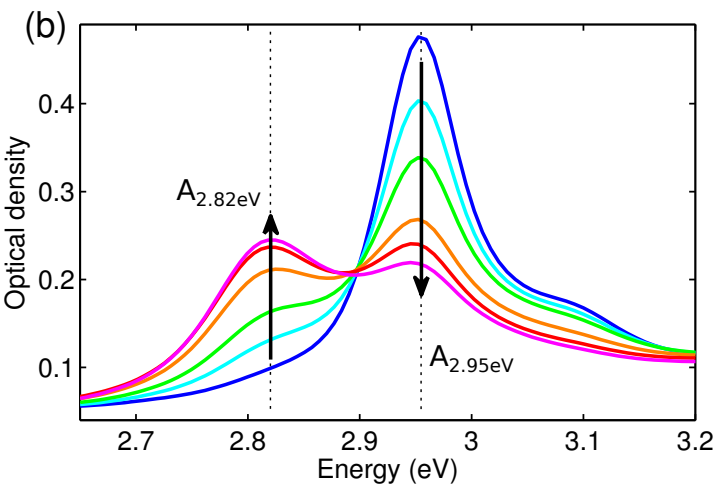

(d)

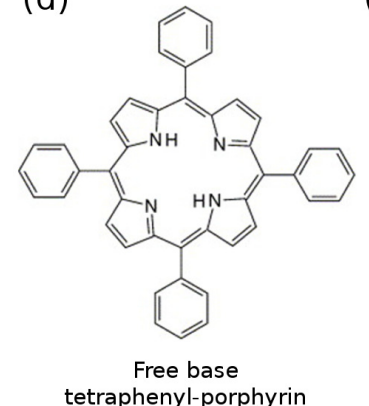

(e)

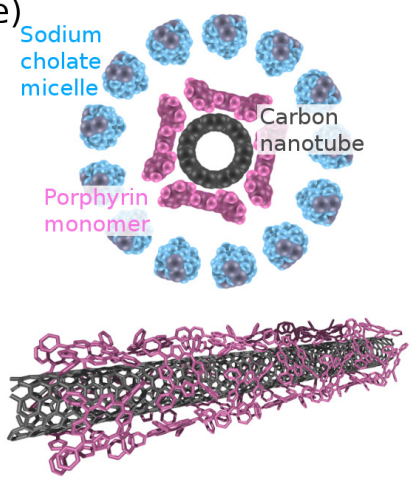

FIG. 1. Kinetics of the adsorption reaction. (a,b) Evolution over time of the absorbance spectrum of an almost stoichiometric mix between micelle wrapped TPP and micelle wrapped HiPCO nanotube suspensions, respectively on the infrared and upper visible range. (c) Evolution over time of the ratio between the absorbance of the peaks relative respectively to the bound and free TPP monomers for a mix left at rest (open dots) or under soft ultrasounds and heating at 40C (red squares). A zoom on the first 6 hours is shown in the inset. The dashed blue line corresponds to the value obtained with the micelle swelling method (see text). (d) chemical sketch of the TPP monomer. (e) Schematic representation of the micelle wrapped functionalized compounds, side and tridimensional views.

resonant response of a specific species that can be identified by the pair of optical transition energies $\left(E_{n, m}^{11}\right.$, $\left.E_{n, m}^{22}\right)$ following the scheme developed in [10]. The functionalization extent of a specific $(n, m)$ species can be deduced from the spectral shifts of its $E_{n, m}^{11}$ and $E_{n, m}^{22}$ lines (Figure $2 \mathrm{~b}$ ).

Another original feature is observed for an excitation energy of $2.85 \mathrm{eV}$, corresponding to the absorption at the Soret Band of adsorbed TPP monomers. In this case, the PL mechanism leading to the emission of the NTs in the near-infrared involves an efficient energy transfer from the TPP to the NT [6]. This transfer is quantitatively assessed through the ratio $R_{n, m}$ between the PL intensities at $E_{n, m}^{11}$ as measured in the case of an excitation through the energy transfer from the TPP molecules and in the case of a direct excitation on the intrinsic $E_{n, m}^{22}$ transition [21].

Figure $3(\mathrm{a}$ and $\mathrm{b})$ exemplifies the spectral shifts of the $E_{n, m}^{11}, E_{n, m}^{22}$ transitions in the particular case of the $(6,5)$ NT species, along with the transfer ratio $R_{6,5}$. All of these three parameters show a similar trend with a threshold and a clear saturation for high TPP concentrations. Actually, these parameters directly reflect the amount of the TPP monomers that are bound to a nanotube. This saturation behavior strongly supports our model where a limited number of adsorbing sites are available on the nanotubes wall which tend to be completely filled at high monomer concentrations. The absence of additional spectroscopic signatures at higher TPP concentration rules out the building of additional layers of TPP. This observation made on the $(6,5)$ species is representative of the observations made for all the other NT species in our sample (see S.I.).

\section{Assessment of the coverage}

To quantitatively evaluate the number of binding sites, and therefore the maximum TPP coverage on the NTs we need to assess the concentration of the NT suspensions. We make use of a $(6,5)$ highly enriched solution (NanoIntegris). Quantitative evaluation of the NT concentration is performed with absorbance measurement (see SI) building on the knowledge of the $E_{6,5}^{22}$ absorption cross-section [21-23].

By monitoring the equilibrium concentration of the 


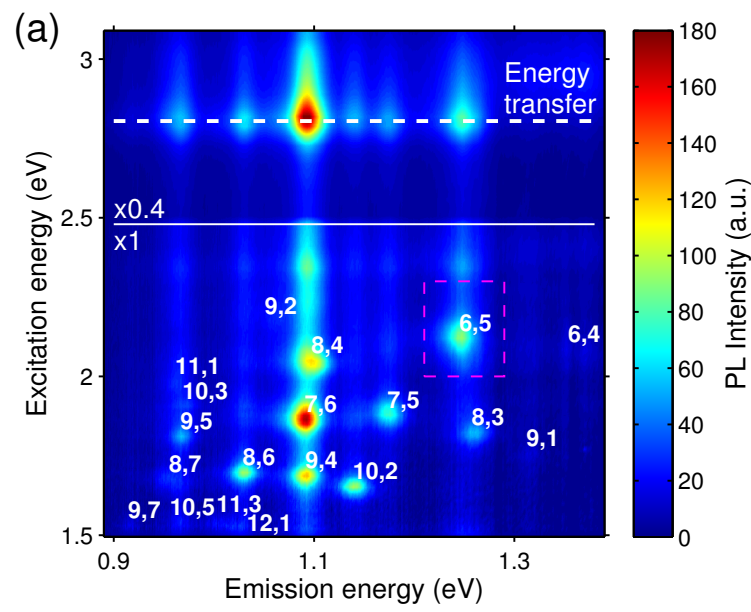

(b)

$[\mathrm{TPP}]_{\text {eq }}=0.15 \mu \mathrm{mol} / \mathrm{L} \quad 0.48 \mu \mathrm{mol} / \mathrm{L}$
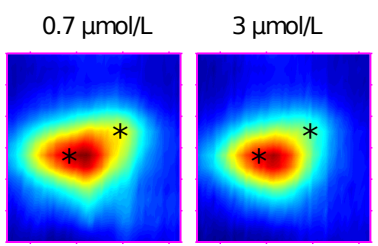

$6.9 \mu \mathrm{mol} / \mathrm{L}$
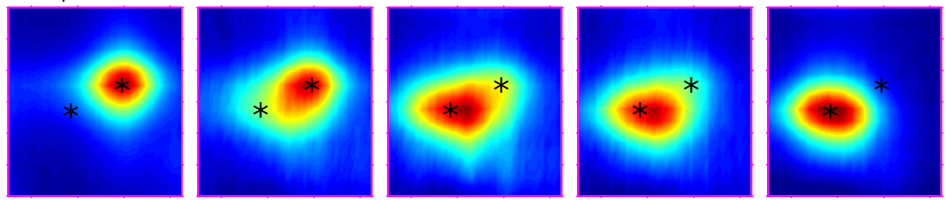

FIG. 2. (a) PLE map of a completely functionalized HiPCO solution. (b) Zoom on the region highlighted by a dashed red rectangle in (a) for selected TPP concentrations illustrating the evolution of the coverage of the $(6,5)$ species. The black stars point at the shifted (resp. unshifted) position of the lines for a fully functionalized (resp. unfunctionalized) sample.
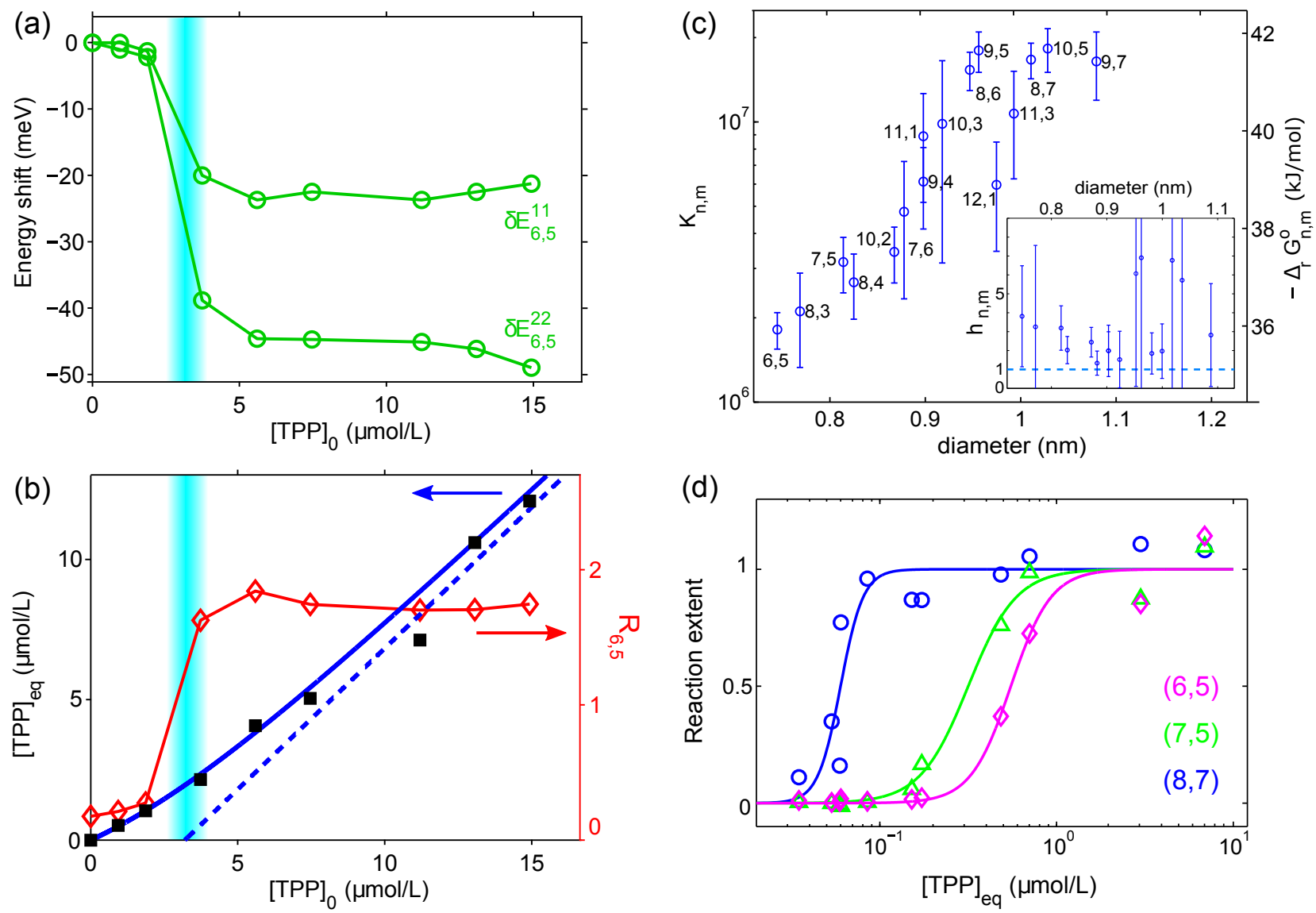

FIG. 3. (a) $E^{11}$ and $E^{22}$ energy shifts (green) as a function of the initial TPP concentration in a highly enriched $(6,5)$ nanotube solution. (b) Free TPP equilibrium concentration (black squares) as a function of the initial TPP concentration, fit of TPP equilibrium concentration to the Hill equation (blue solid line) and its asymptotic form (dashed line); transfer ratio (red, see text) as a function of the initial TPP concentration. The light blue area shows the transition edge of the reaction. (c) Reaction constant (log scale) for several $(n, m)$ nanotube species as a function of the diameter of the species as extracted from the fitting of the data shown in (d). Inset : corresponding Hill coefficients. (d) Functionalization extent as a function the TPP equilibrium concentration (log scale) for selected species (symbols). Solid lines are fits of the Hill equation to these data. 
free TPP in a series of samples made with increasing initial TPP concentration, we observe a nonlinear profile (Figure 3b). At high concentration, a linear asymptote with unitary slope is observed. This observation is typical of a spontaneous total reaction : in a first step, most of the TPP molecules introduced in the solution bind to the NT wall, leaving a limited equilibrium TPP concentration. Once the surface is fully covered, all the additional TPP molecules introduced in the solution remain free, leading to this linear behavior. Therefore, the threshold concentration is directly related to the number of binding sites on the nanotube surface. We note that this threshold concentration is similar to the one observed for the saturation of the energy shifts and of the energy transfer ratio, giving a consistent picture of the reaction (Figure $3(\mathrm{a}$ and $\mathrm{b})$ ). Using the TPP and NT initial concentrations (see S.I.), a fit to the data with the Hill formalism gives a maximum molecular coverage per unit length of nanotube of $N=(2300 \pm 700) \mu \mathrm{m}^{-1}$. This result is compatible with a compact monolayer corresponding to a coverage $N \simeq 1900 \mu \mathrm{m}^{-1}$ (for a $3 \mathrm{TPP}$ ring pattern) or $N \simeq 2500 \mu \mathrm{m}^{-1}$ (for a 4 TPP ring pattern, Figure 1e) and a pitch of $1.6 \mathrm{~nm}$ between two TPP rings along the tube axis (corresponding to the size of the monomer plus the van der Waals distance between the monomers) [7].

\section{DISCUSSION}

\section{Binding energy}

We now turn to the estimate of the reaction constant for each chiral species. To this end, we make use of the spectral shift observed on the $\left(E_{n, m}^{11}, E_{n, m}^{22}\right)$ resonances. The ratio of filled vs total (empty + filled) sites at the surface of a given nanotube species (that is the reaction extent $X_{n, m}$ for this species) is evaluated from the nanotube PL intensities (integrated over a 10x20 meV square window in the $\left(E_{11}, E_{22}\right)$ space) at the shifted and unshifted peaks (Figure $2 \mathrm{~b}$ ). The evolution of the reaction extent as a function of the equilibrium TPP concentration is presented in Figure 3d. The fit of our model to the experimental data gives the reaction constants $K_{n, m}$ and Hill coefficients $h_{n, m}$. The evaluation of these parameters is performed for 16 chiral species. Note that the use of the previously introduced transfer ratio $R_{n, m}$ to monitor the reaction extent for each species gives similar results. However, the former method is more general since it does not rely on the existence of an energy transfer but only on the energy shifts that are systematically induced in functionalization processes. We find Hill coefficients consistently larger than one $\left(<h_{n, m}>\simeq 2.3 \pm 0.8\right)$, meaning that the binding reaction is cooperative and that the transition between pristine and functionalized tubes is steep. This cooperativity may stem from local interac- tions between adsorbed monomers that would ease the formation of a compact monolayer. This is consistent with recent DFT calculations showing a substantial gain in binding energy when monomers form a ring around the nanotube [7].

We obtain large reaction constants $K_{n, m}$ ranging from $2 \times 10^{6}$ to $2 \times 10^{7}$, in good agreement with previously reported values on similar functionalized compounds [2]. These reaction constants are plotted against the species diameter in Figure 3c. This study brings out a strong and monotonic diameter dependence. We find an increase of $K_{n, m}$ of more than a decade for diameters ranging from 0.75 to $1.1 \mathrm{~nm}$. Here again, the actual reaction constant we measure is related to the substitution of cholate by TPP on the nanotube wall. However, it has been established that the affinity of cholate to nanotubes hardly depends on the nanotube diameter $[8,17]$. Therefore, we ascribe this diameter dependence to the $\mathrm{TPP} /$ nanotube interaction. This evolution is interpreted as an increase of the effective $\pi-\pi$ interaction between the aromatic monomer and the nanotube wall when reducing the curvature of the nanotube. We emphasize that this strong species selectivity is observed for small and symmetrical monomers, in contrast to the long polymer chains generally used to yield similar specific species wrapping $[19,24,25]$. Interestingly, this non-covalent affinity trend is in complete opposition with the one observed for the covalent reactivity, which is known to be larger for smaller diameters due to the stronger carbon atom pyramidalization [26].

The reaction constant is associated to a standard Gibbs energy for each chiral species. We observe a monotonic decrease of this Gibbs energy with increasing diameter (Figure 3c) with values ranging from $-36 \mathrm{~kJ} . \mathrm{mol}^{-1}$ for $0.75 \mathrm{~nm}$ diameters down to $-41 \mathrm{~kJ} . \mathrm{mol}^{-1}$ for $1.1 \mathrm{~nm}$ diameters. This Gibbs energy $\Delta_{r} G_{n, m}^{\circ}$ is related to the standard enthalpy $\Delta_{r} H_{n, m}^{\circ}$ and entropy $\Delta_{r} S_{n, m}^{\circ}$ of the reaction through $-R T \ln \left(K_{n, m}\right)=\Delta_{r} G_{n, m}^{\circ}=\Delta_{r} H_{n, m}^{\circ}-$ $T \Delta_{r} S_{n, m}^{\circ}$ where $R=8.3 \mathrm{~J} \cdot \mathrm{mol}^{-1} \cdot \mathrm{K}^{-1}$ is the ideal gas constant and $T$ is the temperature. The microscopic binding energy of a TPP monomer to the nanotube is related to the enthalpy of the reaction which could in principle be assessed by changing the temperature of the reaction. Unfortunately, in the present case, we could not carry out this study on a sufficiently large temperature range, essentially because of the strong sensitivity of the micelle environment to temperature changes (the critical micellar concentration of sodium cholate is indeed changing rapidly in this temperature range [27]).

Theoretical studies have predicted a large binding energy between a TPP monomer and a carbon nanotube, of the order of $100 \mathrm{~kJ} / \mathrm{mol}[7,28]$. This value cannot be directly compared to the experimental Gibbs energy since the entropic term may play a significant role. However, it is interesting to note that both the experimental and theoretical approaches agree on a steady increase with 
the nanotube diameter. In fact, by using a linear fit to the data of Figure 3c, we obtain a variation of the order of $2 \mathrm{~kJ} / \mathrm{mol} / \AA(20 \mathrm{meV} / \AA)$. This value compares well to the change of binding energy obtained from DFT calculations [28] of the order of $40 \mathrm{meV} / \AA$. Therefore, we can deduce that the entropic contribution to the Gibbs energy is either diameter independent or that its variations follow the same trend as for the binding energy. In both cases, the practical consequence is an increased stability of the supramolecular compound for larger diameters.

\section{APPLICATION TO DIAMETER FILTERING}

Such a diameter dependence of the adsorption reaction leads to the preferential functionalization of the larger diameter NTs in a suspension. In addition, the cooperative character of the reaction results in steep transitions when increasing the TPP concentration which leads to quasi sequential functionalization of increasing diameter nanotube species. Here, we show how to make use of this unique property in order to achieve an effective low-pass diameter filtering of an as-grown polydisperse sample. We use the previously described functionalization process on a nanotube suspension showing a broad diameter distribution with a low TPP concentration so that TPP is the limiting reagent. This leads to the selective functionalization of the larger diameter species. The cut-off diameter is directly controlled by the initial TPP concentration. The partially functionalized solution is then centrifuged at $120,000 \mathrm{~g}$ for 5 hours. Due to the slightly lower buoyancy of the functionalized NTs, they tend to sediment at the bottom of the tube leaving a supernatant enriched in the lower diameter species.

As a proof of concept, we apply this protocol to a commercial HiPCO solution and use the PLE resonant intensities to evaluate the species relative abundance : we evaluate the relative suppression of a given species by comparing the integrated PL intensity of this species in the PLE map before and after the sorting process (see SI). Figure 4 shows a relative suppression of up to one order of magnitude for the species with a diameter larger than $0.82 \pm 0.01 \mathrm{~nm}$ for an initial TPP concentration $[T P P]=3 \mu \mathrm{mol} . \mathrm{L}^{-1}$. By tuning the initial TPP concentration to $[T P P]=1 \mu \mathrm{mol} . \mathrm{L}^{-1}$ we are able to bring the diameter edge to $0.87 \pm 0.01 \mathrm{~nm}$. Such a sub-nanometer fine tuning is possible owing to the strong diameter dependence of the reaction constant and to the cooperativity of the reaction $\left(h_{n, m}>1\right)$. We note that the lower diameter nanotubes are not functionalized in the end of the process, which can be valuable for applications targeting pristine nanotubes. Reciprocally, the larger diameter fraction consists of functionalized nanotubes. However, in contrast to alternative approaches obtained by selective wrapping of NTs with polymeric surfactants such as DNA [24] or PFO chains [25], our protocol allows an

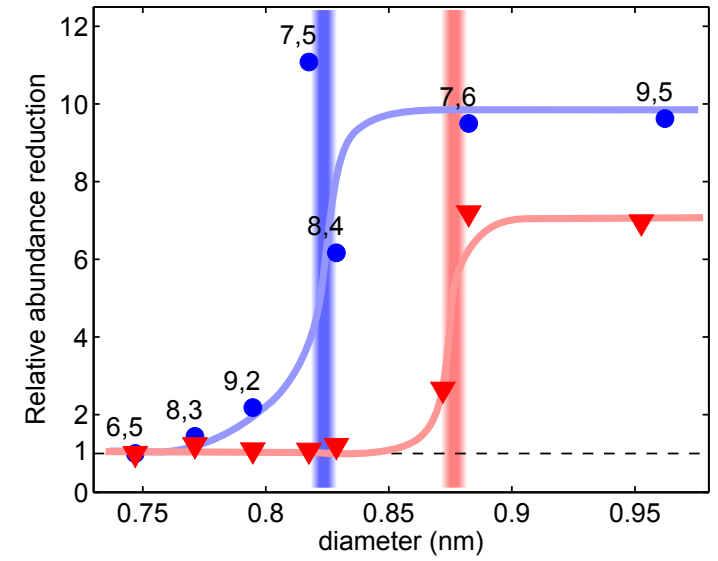

FIG. 4. Relative reduction of the resonant PL intensity of different species, between a stock HiPCO solution and the same solution after partial functionalization followed by a 5 hour centrifugation (120000 g). Two different monomers concentrations $\left(1 \mu \mathrm{mol} . \mathrm{L}^{-1}\right.$, red or $3 \mu \mathrm{mol} . \mathrm{L}^{-1}$, blue) give different diameter selectivity. The vertical lines highlight the cut-off diameter for each TPP concentration.

easy recovery of the pristine chirality enriched NTs using a regular rinsing process on PTFE filters, due to the high solubility of TPP monomers in organic solvents.

\section{CONCLUSION}

In conclusion, we have shown that the non-covalent functionalization of the sidewall of nanotubes with TPP monomers yields a single layer structure with a higher affinity for larger diameter nanotubes. We have gained insight into the microscopic mechanisms at stake in the synthesis of noncovalent carbon-based hybrid compounds through thermodynamical monitoring of the reaction. Finally, we demonstrated an original scheme for diameter filtering of polydisperse nanotube samples by making use of this chemical selectivity. We achieved a fine control over the target diameter simply by adjusting the TPP concentration. Further optimization of the process may pave the way to large scale efficient diameter sorting of nanotubes species for opto-electronic applications targeting a specific working wavelength.

[1] C. Ehli, C. Oelsner, D. M. Guldi, A. Mateo-Alonso, M. Prato, C. Schmidt, C. Backes, F. Hauke, and A. Hirsch, Nat Chem 1, 243 (2009).

[2] J. K. Sprafke, S. D. Stranks, J. H. Warner, R. J. Nicholas, and H. L. Anderson, Angewandte Chemie International Edition 50, 2313 (2011).

[3] F. Ernst, T. Heek, A. Setaro, R. Haag, and S. Reich, Advanced Functional Materials 22, 3921 (2012). 
[4] M. S. Arnold, J. L. Blackburn, J. J. Crochet, S. K. Doorn, J. G. Duque, A. Mohite, and H. Telg, Phys. Chem. Chem. Phys. 15, 14896 (2013).

[5] C. Roquelet, B. Langlois, F. Vialla, D. Garrot, J. Lauret, and C. Voisin, Chemical Physics 413, 45 (2013).

[6] C. Roquelet, D. Garrot, J. S. Lauret, C. Voisin, V. AlainRizzo, P. Roussignol, J. A. Delaire, and E. Deleporte, Appl. Phys. Lett. 97, 141918 (2010).

[7] W. Orellana, Applied Physics Letters 105, 023110 (2014).

[8] Y. Kato, A. Inoue, Y. Niidome, and N. Nakashima, Scientific reports 2 (2012).

[9] M. J. Shea, R. D. Mehlenbacher, M. T. Zanni, and M. S. Arnold, The Journal of Physical Chemistry Letters, J. Phys. Chem. Lett. 5, 3742 (2014).

[10] S. M. Bachilo, M. S. Strano, C. Kittrell, R. H. Hauge, R. E. Smalley, and R. B. Weisman, Science 298, 2361 (2002).

[11] W. Wenseleers, I. Vlasov, E. Goovaerts, E. Obraztsova, A. Lobach, and A. Bouwen, Advanced Functional Materials 14, 1105 (2004).

[12] S. Lin and D. Blankschtein, The Journal of Physical Chemistry B 114, 15616 (2010).

[13] C. Roquelet, J. S. Lauret, V. Alain-Rizzo, C. Voisin, R. Fleurier, M. Delarue, D. Garrot, A. Loiseau, P. Roussignol, J. A. Delaire, and E. Deleporte, ChemPhysChem 11, 1667 (2010).

[14] G. Magadur, J.-S. Lauret, V. Alain-Rizzo, C. Voisin, P. Roussignol, E. Deleporte, and J. A. Delaire, ChemPhysChem 9, 1250 (2008).

[15] S. Berger, F. Iglesias, P. Bonnet, C. Voisin, G. Cassabois, J. S. Lauret, C. Delalande, and P. Roussignol, Journal of Applied Physics 105, 094323 (2009).

[16] J. Leng, S. U. Egelhaaf, and M. E. Cates, Biophysical Journal 85, 1624 (2003).

[17] T. J. McDonald, C. Engtrakul, M. Jones, G. Rumbles, and M. J. Heben, The Journal of Physical Chemistry B, J. Phys. Chem. B 110, 25339 (2006).

[18] J. N. Weiss, The FASEB Journal 11, 835 (1997).

[19] S.-Y. Ju, J. Doll, I. Sharma, and F. Papadimitrakopoulos, Nature nanotechnology 3, 356 (2008).

[20] H. Oh, J. Sim, and S.-Y. Ju, Langmuir 29, 11154 (2013).

[21] F. Vialla, C. Roquelet, B. Langlois, G. Delport, S. M. Santos, E. Deleporte, P. Roussignol, C. Delalande, C. Voisin, and J.-S. Lauret, Phys. Rev. Lett. 111, 137402 (2013).

[22] L. Oudjedi, A. N. G. Parra-Vasquez, A. G. Godin, L. Cognet, and B. Lounis, The Journal of Physical Chemistry Letters 4, 1460 (2013).

[23] J. K. Streit, S. M. Bachilo, S. Ghosh, C.-W. Lin, and R. B. Weisman, Nano Letters, Nano Lett. 14, 1530 (2014).

[24] M. Zheng, A. Jagota, M. S. Strano, A. P. Santos, P. Barone, S. G. Chou, B. A. Diner, M. S. Dresselhaus, R. S. Mclean, G. B. Onoa, G. G. Samsonidze, E. D. Semke, M. Usrey, and D. J. Walls, Science 302, 1545 (2003).

[25] A. Nish, J.-Y. Hwang, J. Doig, and R. J. Nicholas, Nature Nanotechnology 2, 640 ((2007)).

[26] Z. Chen, W. Thiel, and A. Hirsch, ChemPhysChem 4, 93 (2003).

[27] A. Maestre, P. Guardado, and M. Moyá, J. Chem. Eng. Data 59, 433 (2014).

[28] W. Orellana, J. Mater. Sci. 50, 898 (2015). 